\title{
SUPPORTING INFORMATION \\ 1D to 3D and Chiral to Noncentrosymmetric Metal-Organic \\ Complexes Controlled by the Amount of DEF Solvent: \\ Photoluminescent and NLO Properties
}

Yuehong Wen, Tianlu Sheng, Chao Zhuo, Xiaoquan Zhu, Shengmin Hu, Wenhai Cao, Haoran Li,

Hao Zhang, and Xintao $\mathrm{Wu}^{*}$

State Key Laboratory of Structure Chemistry Fujian Institute of Research on the Structure of

Matter, Chinese Academy of Sciences, Fuzhou, 350002, China.

E-mail: wxt@fjirsm.ac.cn; Tel: +86-591-63179449 
Table S1 Selected bond lengths $(\AA)$ and angles $\left(^{\circ}\right)$ of complexes 1-3.

Table S1.1 Selected bond lengths $(\AA)$ and angles $\left({ }^{\circ}\right)$ of complex $\mathbf{1 b}$.

\begin{tabular}{llll}
\hline $\operatorname{Zn}(1)-\mathrm{N}(3)^{\mathrm{a}}$ & $2.121(6)$ & $0(1)-\mathrm{Zn}(1)-\mathrm{N}(1)$ & $93.3(2)$ \\
$\mathrm{Zn}(1)-0(1)$ & $2.155(6)$ & $\mathrm{N}(4)^{\mathrm{b}}-\mathrm{Zn}(1)-\mathrm{N}(1)$ & $92.7(2)$ \\
$\mathrm{Zn}(1)-\mathrm{N}(4)^{\mathrm{b}}$ & $2.182(6)$ & $\mathrm{N}(3)^{\mathrm{a}}-\mathrm{Zn}(1)-\mathrm{N}(2)$ & $95.5(2)$ \\
$\mathrm{Zn}(1)-\mathrm{N}(1)$ & $2.185(6)$ & $0(1)-\mathrm{Zn}(1)-\mathrm{N}(2)$ & $92.5(2)$ \\
$\mathrm{Zn}(1)-\mathrm{N}(2)$ & $2.190(6)$ & $\mathrm{N}(4)^{\mathrm{b}}-\mathrm{Zn}(1)-\mathrm{N}(2)$ & $173.6(3)$ \\
$\mathrm{Zn}(1)-0(2)$ & $2.255(5)$ & $\mathrm{N}(1)-\mathrm{Zn}(1)-\mathrm{N}(2)$ & $81.9(3)$ \\
$\mathrm{N}(3)-\mathrm{Zn}(1)^{\mathrm{c}}$ & $2.121(6)$ & $\mathrm{N}(3)^{\mathrm{a}}-\mathrm{Zn}(1)-0(2)$ & $89.7(2)$ \\
$\mathrm{N}(4)-\mathrm{Zn}(1)^{\mathrm{d}}$ & $2.182(6)$ & $0(1)-\mathrm{Zn}(1)-0(2)$ & $178.2(3)$ \\
$\mathrm{N}(3)^{\mathrm{a}}-\mathrm{Zn}(1)-0(1)$ & $91.9(2)$ & $\mathrm{N}(4)^{\mathrm{b}}-\mathrm{Zn}(1)-0(2)$ & $89.7(2)$ \\
$\mathrm{N}(3)^{\mathrm{a}}-\mathrm{Zn}(1)-\mathrm{N}(4)^{\mathrm{b}}$ & $89.6(3)$ & $\mathrm{N}(1)-\mathrm{Zn}(1)-0(2)$ & $85.2(2)$ \\
$0(1)-\mathrm{Zn}(1)-\mathrm{N}(4)^{\mathrm{b}}$ & $91.3(2)$ & $\mathrm{N}(2)-\mathrm{Zn}(1)-0(2)$ & $86.5(2)$ \\
$\mathrm{N}(3)^{\mathrm{a}}-\mathrm{Zn}(1)-\mathrm{N}(1)$ & $174.3(3)$ & &
\end{tabular}

Symmetry codes: (a) x, 2-y, z+1/2; (b) x, 1-y, z+1/2; (c) x, 2-y, z-1/2; (d) x, 1-y, z-1/2.

Table S1.2 Selected bond lengths $(\AA)$ and angles $\left({ }^{\circ}\right)$ of complex 2.

\begin{tabular}{llll}
\hline $\mathrm{Zn}(1)-0(2)$ & $2.100(2)$ & $\mathrm{N}(4)^{\mathrm{a}}-\mathrm{Zn}(1)-0(3)$ & $90.14(9)$ \\
$\mathrm{Zn}(1)-\mathrm{N}(4)^{\mathrm{a}}$ & $2.133(2)$ & $0(1)-\mathrm{Zn}(1)-0(3)$ & $87.88(8)$ \\
$\mathrm{Zn}(1)-0(1)$ & $2.141(2)$ & $0(2)-\mathrm{Zn}(1)-\mathrm{N}(1)$ & $88.01(8)$ \\
$\mathrm{Zn}(1)-0(3)$ & $2.167(2)$ & $\mathrm{N}(4)^{\mathrm{a}}-\mathrm{Zn}(1)-\mathrm{N}(1)$ & $176.27(10)$ \\
$\mathrm{Zn}(1)-\mathrm{N}(1)$ & $2.174(2)$ & $0(1)-\mathrm{Zn}(1)-\mathrm{N}(1)$ & $86.29(8)$ \\
$\mathrm{Zn}(1)-\mathrm{N}(2)$ & $2.261(2)$ & $0(3)-\mathrm{Zn}(1)-\mathrm{N}(1)$ & $93.57(8)$ \\
$\mathrm{N}(4)-\mathrm{Zn}(1)^{\mathrm{b}}$ & $2.133(2)$ & $0(2)-\mathrm{Zn}(1)-\mathrm{N}(2)$ & $91.11(8)$ \\
$0(2)-\mathrm{Zn}(1)-\mathrm{N}(4)^{\mathrm{a}}$ & $92.56(8)$ & $\mathrm{N}(4)^{\mathrm{a}}-\mathrm{Zn}(1)-\mathrm{N}(2)$ & $94.59(9)$ \\
$0(2)-\mathrm{Zn}(1)-0(1)$ & $172.23(8)$ & $0(1)-\mathrm{Zn}(1)-\mathrm{N}(2)$ & $93.30(8)$ \\
$\mathrm{N}(4)^{\mathrm{a}}-\mathrm{Zn}(1)-0(1)$ & $93.47(8)$ & $0(3)-\mathrm{Zn}(1)-\mathrm{N}(2)$ & $175.05(8)$ \\
$0(2)-\mathrm{Zn}(1)-0(3)$ & $87.19(8)$ & $\mathrm{N}(1)-\mathrm{Zn}(1)-\mathrm{N}(2)$ & $81.72(8)$ \\
\hline
\end{tabular}

Symmetry codes: (a) $\mathrm{x}, \mathrm{y}, \mathrm{z}+1$; (b) $\mathrm{x}, \mathrm{y}, \mathrm{z}-1$.

Table S1.3 Selected bond lengths $(\AA)$ and angles $\left({ }^{\circ}\right)$ of complex 3.

\begin{tabular}{llll}
\hline $\mathrm{Zn}(1)-\mathrm{N}(3)^{\mathrm{a}}$ & $2.146(4)$ & $0(2)-\mathrm{Zn}(1)-0(1)$ & $174.22(12)$ \\
$\mathrm{Zn}(1)-0(2)$ & $2.148(3)$ & $\mathrm{N}(1)-\mathrm{Zn}(1)-0(1)$ & $90.44(13)$ \\
$\mathrm{Zn}(1)-\mathrm{N}(1)$ & $2.152(4)$ & $\mathrm{N}(3)^{\mathrm{a}}-\mathrm{Zn}(1)-\mathrm{N}(4)^{\mathrm{b}}$ & $91.40(13)$ \\
$\mathrm{Zn}(1)-\mathrm{N}(4)^{\mathrm{b}}$ & $2.202(3)$ & $0(2)-\mathrm{Zn}(1)-\mathrm{N}(4)^{\mathrm{b}}$ & $89.88(13)$ \\
$\mathrm{Zn}(1)-0(1)$ & $2.208(3)$ & $\mathrm{N}(1)-\mathrm{Zn}(1)-\mathrm{N}(4)^{\mathrm{b}}$ & $92.12(13)$ \\
$\mathrm{Zn}(1)-\mathrm{N}(2)$ & $2.258(3)$ & $0(1)-\mathrm{Zn}(1)-\mathrm{N}(4)^{\mathrm{b}}$ & $95.02(14)$ \\
$\mathrm{N}(3)-\mathrm{Zn}(1)^{\mathrm{c}}$ & $2.146(4)$ & $\mathrm{N}(3)^{\mathrm{a}}-\mathrm{Zn}(1)-\mathrm{N}(2)$ & $95.22(12)$ \\
$\mathrm{N}(4)-\mathrm{Zn}(1)^{\mathrm{d}}$ & $2.208(3)$ & $0(2)-\mathrm{Zn}(1)-\mathrm{N}(2)$ & $85.78(13)$ \\
$\mathrm{N}(3)^{\mathrm{a}}-\mathrm{Zn}(1)-0(2)$ & $91.45(13)$ & $\mathrm{N}(1)-\mathrm{Zn}(1)-\mathrm{N}(2)$ & $81.56(12)$ \\
$\mathrm{N}(3)^{\mathrm{a}}-\mathrm{Zn}(1)-\mathrm{N}(1)$ & $174.75(16)$ & $0(1)-\mathrm{Zn}(1)-\mathrm{N}(2)$ & $89.69(13)$ \\
$0(2)-\mathrm{Zn}(1)-\mathrm{N}(1)$ & $92.44(13)$ & $\mathrm{N}(4)^{\mathrm{b}}-\mathrm{Zn}(1)-\mathrm{N}(2)$ & $172.17(13)$ \\
\hline & & $\mathrm{S} 2$
\end{tabular}




$$
\mathrm{N}(3)^{\mathrm{a}}-\mathrm{Zn}(1)-0(1) \quad 85.38(13)
$$

Symmetry codes: (a) $\mathrm{x},-\mathrm{y}, \mathrm{z}-1 / 2$; (b) $\mathrm{x}-1 / 2, \mathrm{y}-1 / 2$, z; (c) $\mathrm{x},-\mathrm{y}, \mathrm{z}+1 / 2$; (d) $\mathrm{x}+1 / 2, \mathrm{y}+1 / 2$, z.

Figure S1 (a) The interlayer distance of $\mathbf{1 b}$. (b) The $\pi$ - $\pi$ interactions.

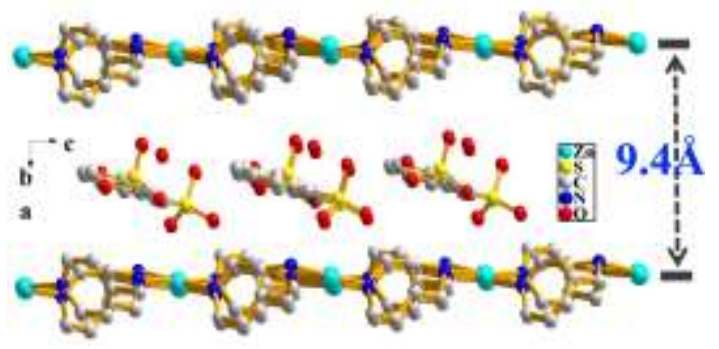

(a)

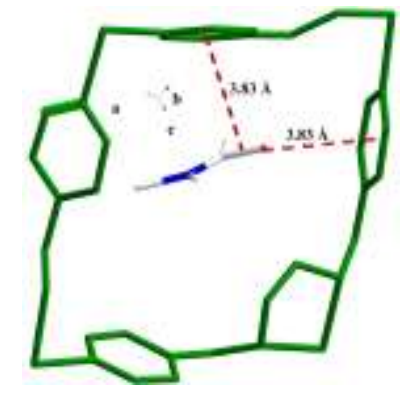

(b)

Figure S2 (a) Hydrogen bonds existed in 2 and 2D layers composed of G1 viewed down the $c$ axis. (b) $\pi-\pi$ interactions. (c) Solid-state $C D$ spectra of samples 2 and $\mathbf{2 X}$.

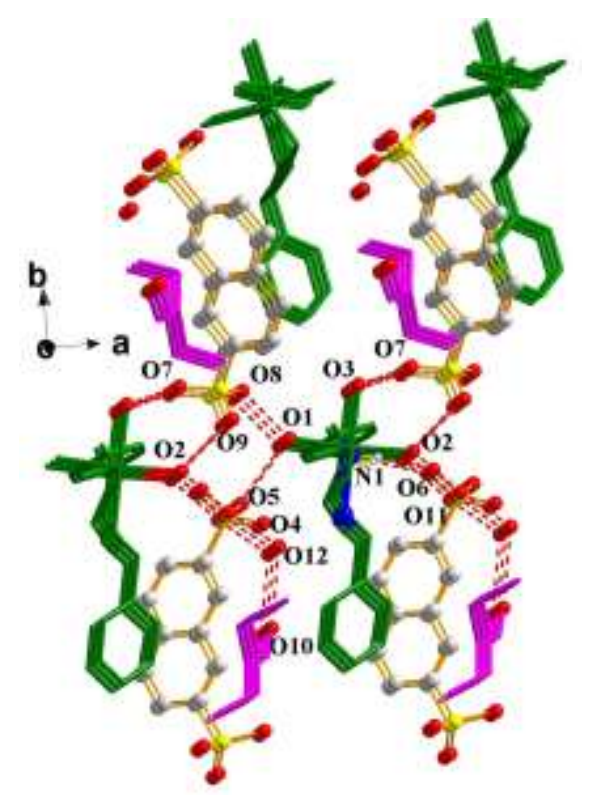


(a)

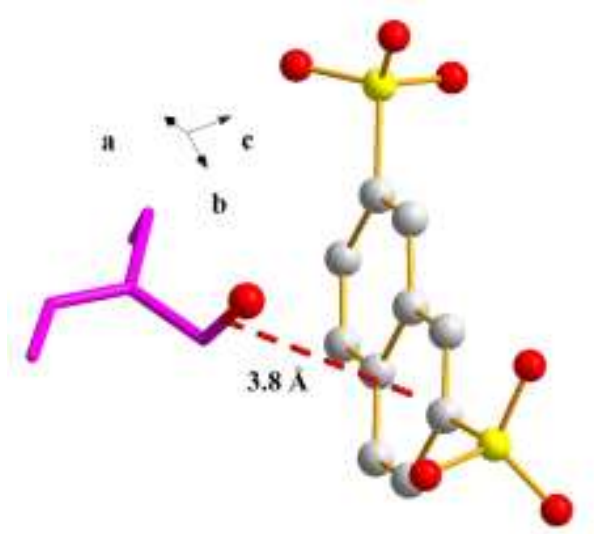

(b)

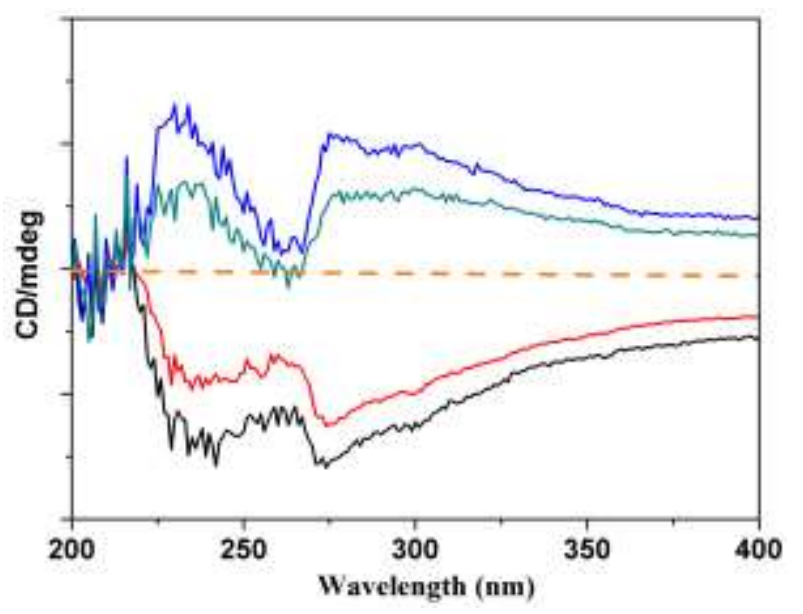

(c)

Figure S3 (a) The arrangements of G1 in MOC 3 viewed down the a axis. (b) 3D Structure composed of G1 viewed down the c axis. 


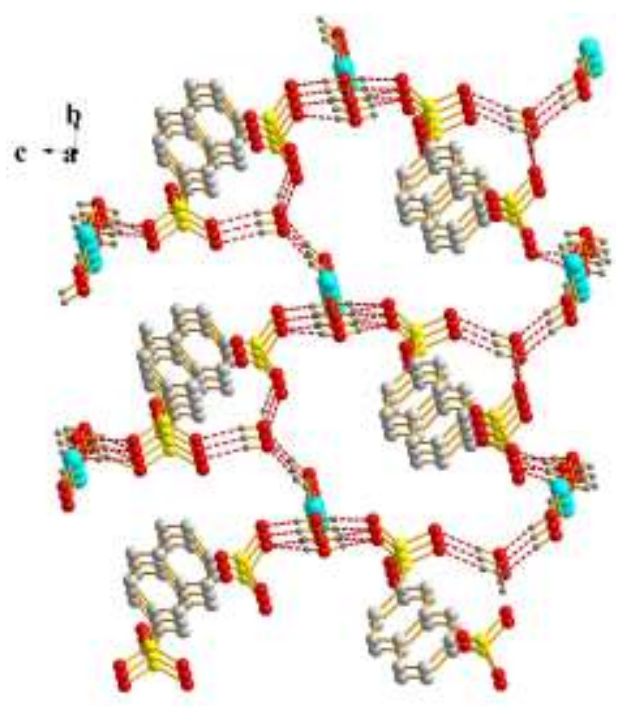

(a)

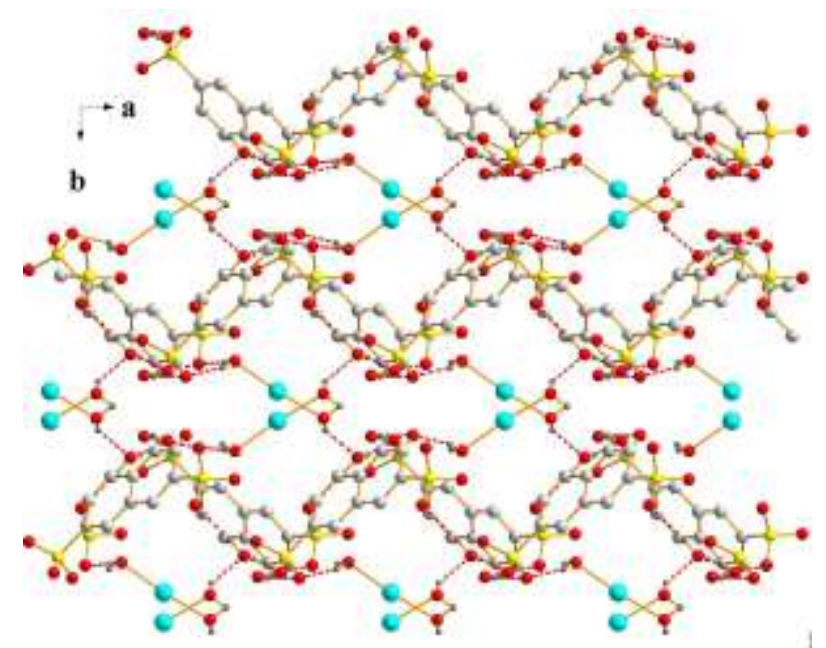

(b)

Figure S4 Photographic images of compounds $\mathbf{1 a}(a)$ and $\mathbf{2}(b)$.

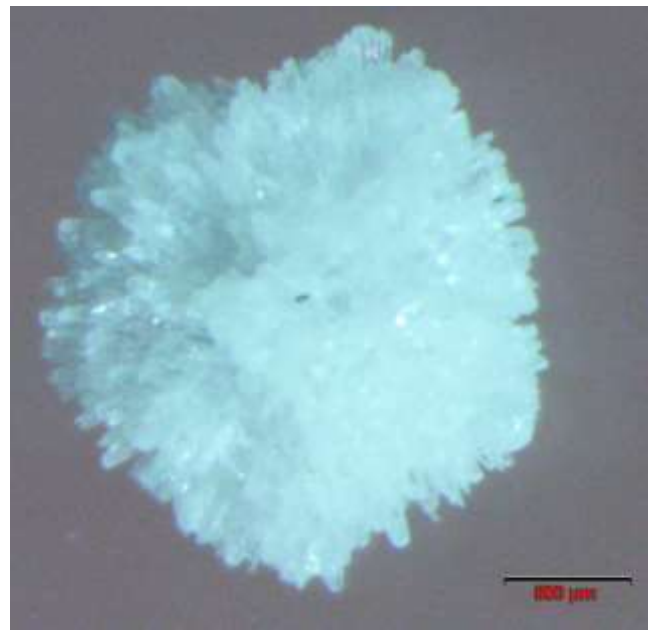

(a) 


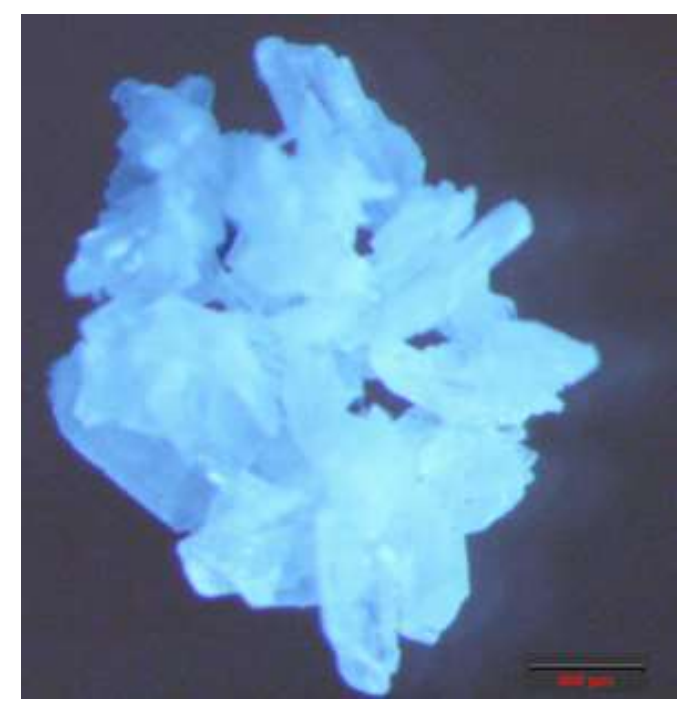

(b)

Figure S5 PXRD patterns for complex 1.

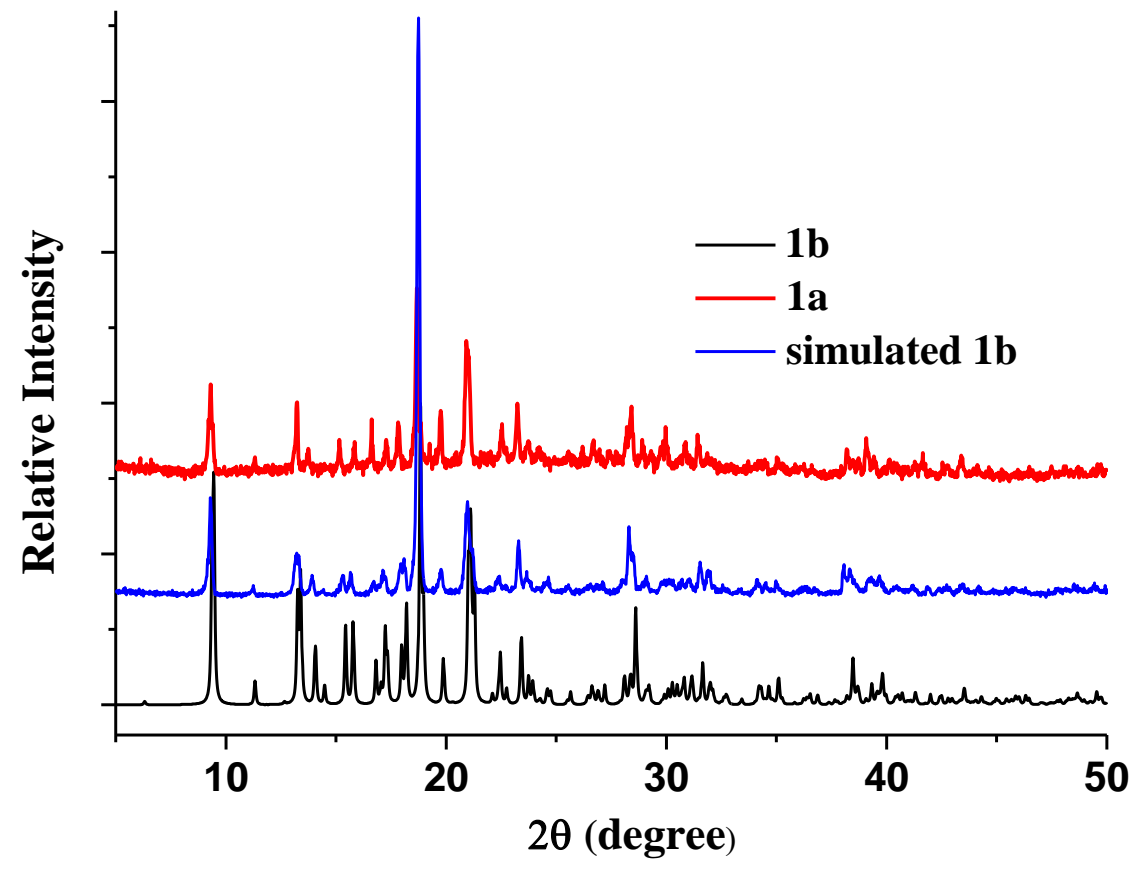

Figure S6 PXRD patterns for complex 2. 


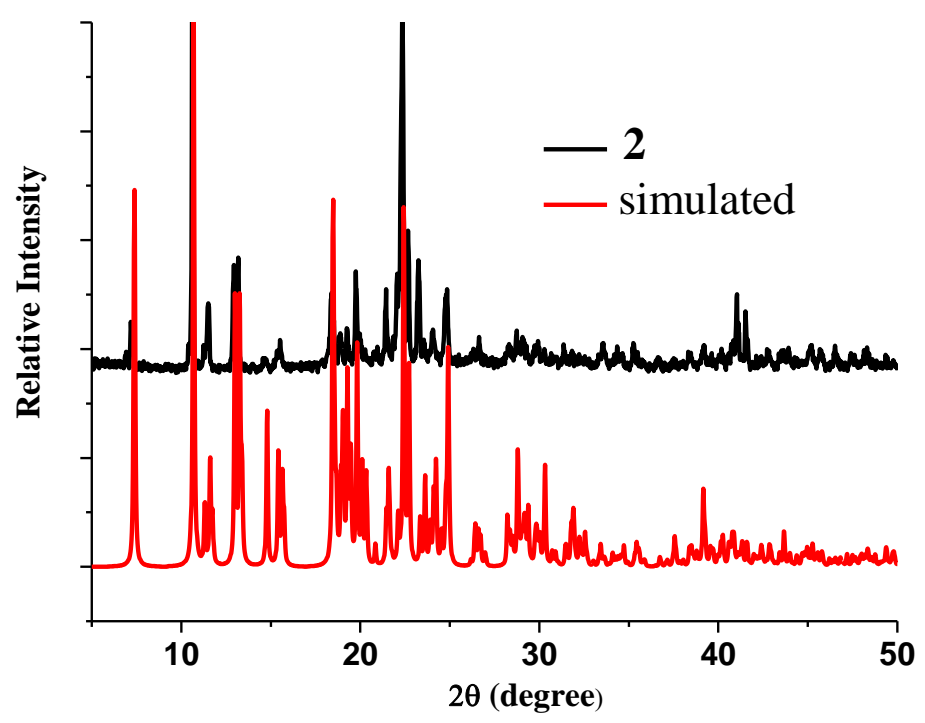

Figure S7 PXRD patterns for complex 3.

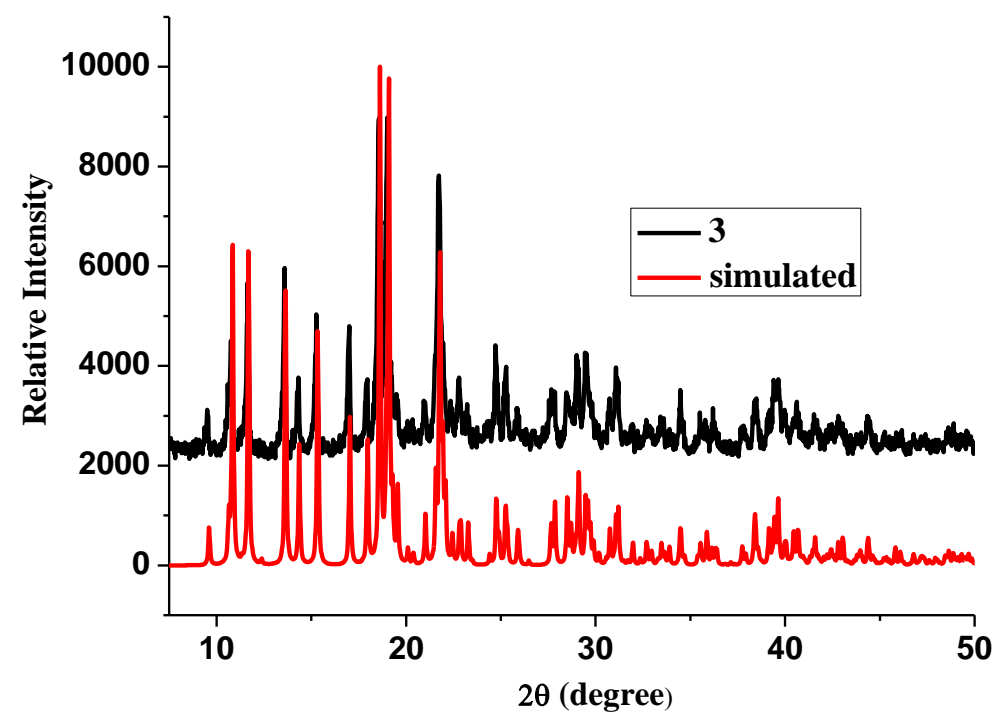

Figure $S 8$ TGA plots for $\mathbf{1 a}$. 


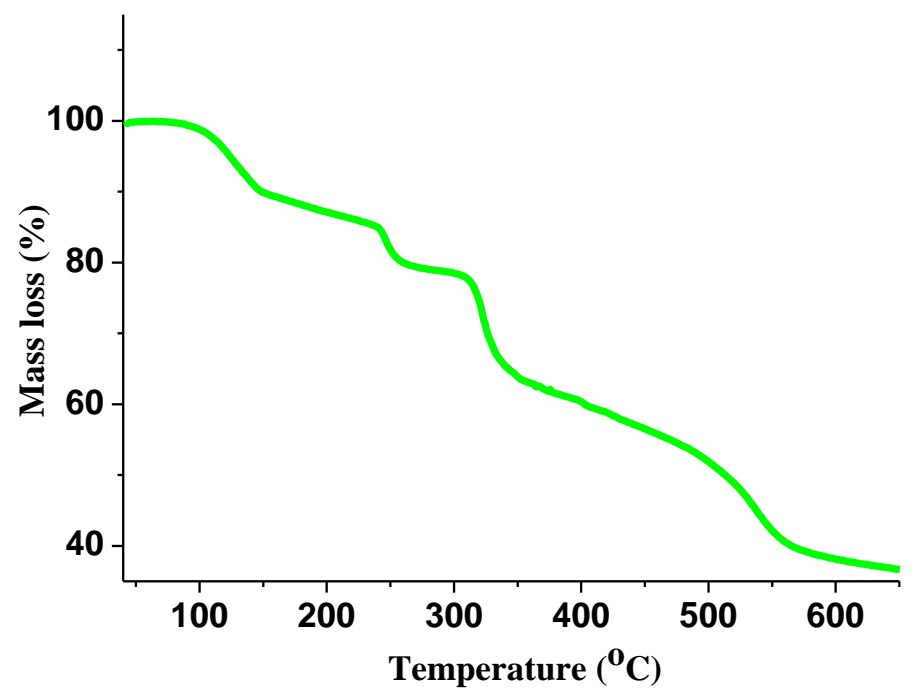

Figure $\mathbf{S 9}$ TGA plots for $\mathbf{1 b}$.

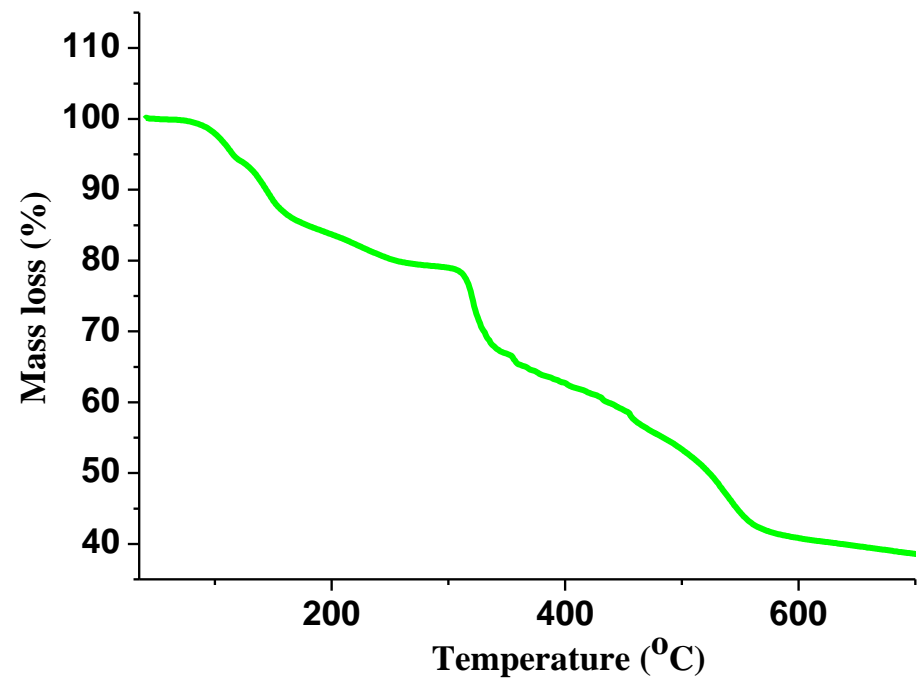

Figure S10 TGA plots for 2. 


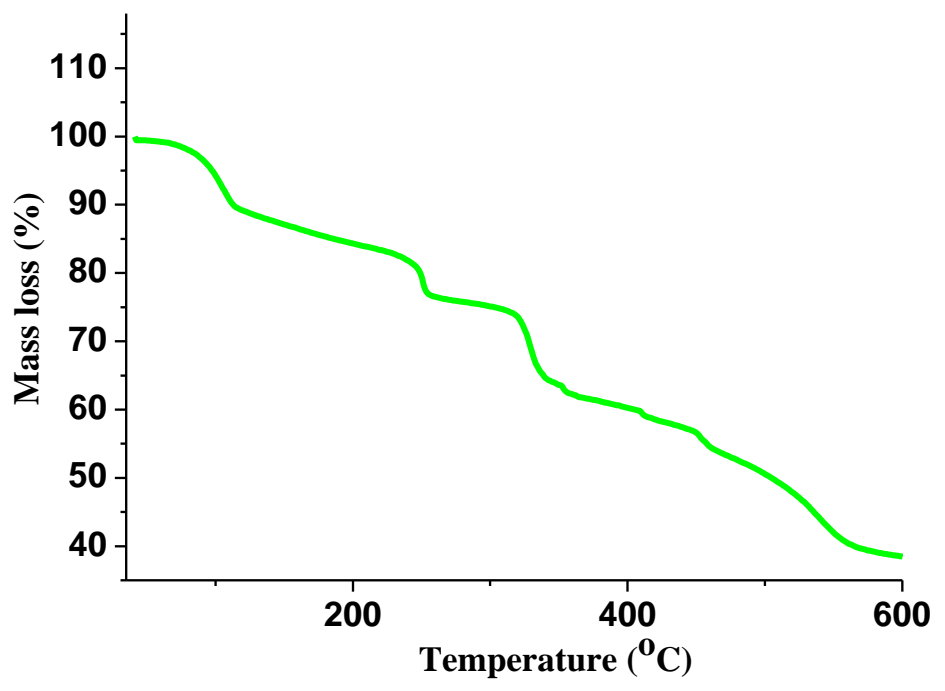

Figure S11 TGA plots for 3 .

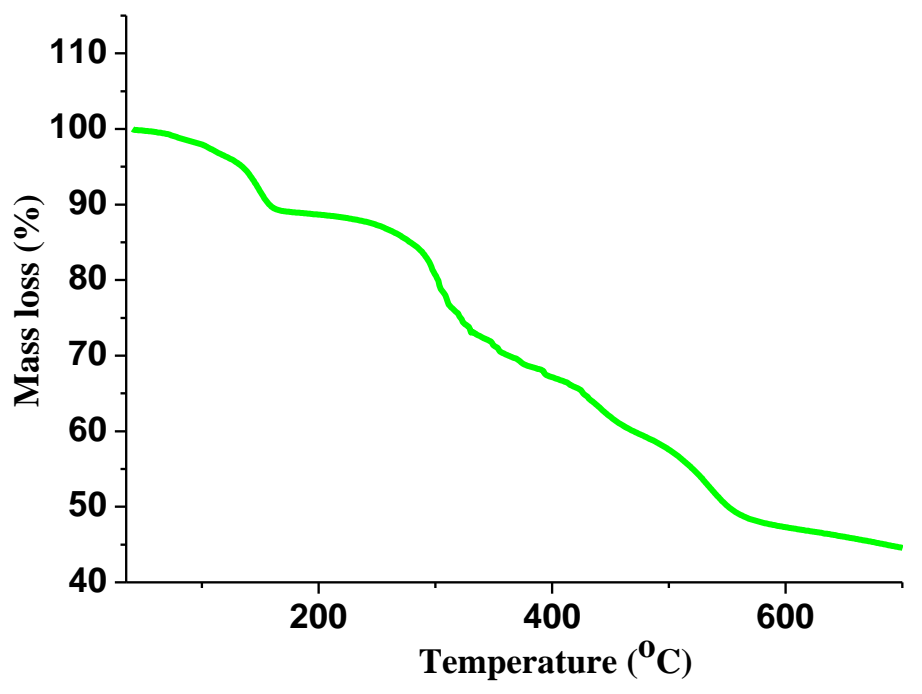

